\title{
ON THE DISTRIBUTION OF LUMINOUS MATTER IN A FRIEDMANN
} UNIVERSE

\author{
D.S. Wamalwa \\ University of Nairobi, Department of Physics, Astronomy and Astrophysics Group, P.O. Box 30197-00100, \\ Nairobi. \\ dismasw@yahoo.com
}

\begin{abstract}
The Friedmann model is considered through stereographic projection of the Friedmann-Roberson-Walker metric for open, flat and closed matter-dominated universe. A relation between redshift and number density of galaxies is derived on the platform of this metric while neglecting the effects of dark matter and dark energy. The derived relation is observed to have the potential to test the fractal-homogeneous nature of our universe based on accurate future observational data.
\end{abstract}

\section{Indexing terms/Keywords}

Galaxies, Redshift, Number density, Friedmann, Fractal

\section{Academic Discipline And Sub-Disciplines}

Astrophysics.

\section{SUBJECT CLASSIFICATION}

AMS Subject classification: 83,85

\section{INTRODUCTION}

The orthodox picture of an isotropic and homogeneous universe (Friedmann universe) as strongly supported by the cosmic microwave background radiation [1] and the cosmological principle is at stake today as three-dimensional redshift surveys prober deeper into the universe uncovering more inhomogeneous structures (fractal) on large length scales [2]. The existence of structures like galaxies in clusters and voids is believed to point towards inhomogeneities and therefore, raises the question as to whether or not visible matter distribution really becomes homogeneous on certain length scale or if the fractal model is as a result of misinterpretations of the limited available observational data of the universe.

The three-dimensional maps depicting inhomogeneities (fractal) and showing no clear tendency to homogenization (Friedmann) even on largest length scales, however, depend on our questionable ability to perform accurate distance measurements $[3,4,5,6]$. A theoretically elegant approach to ruling out the homogeneous distribution of visible matter in accordance with the Friedmann universe is firstly to consider the inter-relationship between measured astronomical objects that involve no assumptions about the background geometry; and then secondly, prepare the results for comparison with observed dependencies. In earlier work [7], the first part of this problem was addressed by deriving light intensity-redshift relation of galaxies. In the present work, we present an alternative approach to this problem by investigating the relationship between redshift and number density of galaxies based on the Friedmann metric, ignoring the effects of dark matter and dark energy. Therefore, the fundamental aspect of this paper is to derive the relationship between measured redshift, $z$ and number density of galaxies, $n(z)$, from data that does not involve distance measurements. It turns out that the present derived relation is a suitable candidate for judgement of a fractal-Friedmann universe. Furthermore, it has the necessary ingredients to judge the nature of the universe i.e., whether elliptic, flat or hyperbolic.

In subsequent work, we intend to address the second part of the problem by performing simulations of the derived light intensity-redshift relation and number density-redshift relation. Completion of the second part of the problem will put us in a position to judge with certainty as to whether or not our universe is indeed Friedmann on any given length scale.

\section{FRACTAL MODEL}

According to Charliers's concept of hierarchical clustering, fractals are systems in which more and more structures appear at smaller and smaller scales and the structures at small scales are similar to those at large scales [8, 9, 10]. The fractal dimension, $D$, is a measure of the strength of singularity around the structure points given as:

$$
N(r)=B r^{D},
$$

where the prefactor $B=N_{0} / r_{0}^{D} ; N_{0}$ is the number of points within a length scale $r_{0}$ while $D=3$ and $D<3$ implies homogeneous and fractal systems respectively.

Nevertheless, fractal systems are incompatible with two-point correlation function that is instrumental in analysis of redshift catalogue data. This is because fractal systems have no defined average density between lower and upper cutoffs as they lack upper cut-off limit. Furthermore, the apriori assumption in cosmology that the average density must tend to a finite 
non-zero value embraces the homogeneity hypothesis [11]. Therefore, it is possible that the fractal nature of the universe as claimed is due to misinterpretation of available observational data.

\section{FRIEDMANN MODEL}

The Friedmann model describes an isotropic, homogeneous and expanding universe [12]. The model embodies the cosmological principle in which there is a preferred time coordinate $t$ such that the $t=$ constant slices are homogeneous and isotropic spaces: the sphere (constant positive curvature), flat space (no curvature), and hyperbolic space (constant negative curvature). The use of stereographic projection in rectangular coordinate system on the three-dimensional projection space handles the three cases altogether [7]. In this case, the Friedmann metric becomes:

$$
g=c^{2} d t^{2}-\frac{R(t)^{2}}{\left(1+\kappa r^{2}\right)^{2}}\left(d x^{2}+d y^{2}+d z^{2}\right)
$$

where $r^{2}=x^{2}+y^{2}+z^{2} ; \kappa=1,0,-1$ for the spherical, flat, hyperbolic cases respectively; $R(t)$ is the cosmic scale factor (the radius of the universe at time $t$ for the case of the unit sphere when $\kappa=1$ ); and $c$ is the speed of light. In spherical coordinates, on the projection space, the metric reads:

$$
g=c^{2} d t^{2}-\frac{R(t)^{2}}{\left(1+\kappa r^{2}\right)^{2}}\left(d r^{2}+r^{2} d \theta^{2}+r^{2} \sin ^{2} \theta d \varphi^{2}\right)
$$

\subsection{Field Equations and Conservation Law}

Einstein field equations for describing dynamics and evolution of the universe based on the Friedmann model have already been shown [7] to be:

$$
12 \kappa c^{2}+3 R^{\prime}(t)^{2}=B c^{4} R(t)^{2} \rho(t)
$$

and

$$
4 \kappa^{2} c^{2}+2 R(t) R^{\prime \prime}(t)+R^{\prime}(t)^{2}=-B c^{2} R(t)^{2} p(t)
$$

where

$$
R^{\prime}(t)=\frac{d R}{d t} ; \quad R^{\prime \prime}(t)=\frac{d^{2} R}{d t^{2}} ; \quad B=8 \pi G c^{-4}
$$

$\rho(t)$ is the mass density of matter in the universe at time $t ; \rho(t)$ is the pressure of matter in the universe at time $t$; and $G$ is the gravitational constant.

Furthermore, a conservation law based on equations (3) and (4) for a pressureless, matter-dominated, universe has been written down as [7]:

$$
d t=\frac{d R}{\sqrt{\frac{B c^{2} \alpha}{3 R}}-4 \kappa c^{2} R} ; \quad \alpha=\rho(t) R(t)^{3}
$$

where $\alpha$ is a constant.

\section{RELATION BETWEEN NUMBER DENSITY OF GALAXIES AND REDSHIFT}

Suppose we are given a large list of all astronomical objects that involve no assumptions about the background geometry for which the redshift, $z$ of light and the number density, $n(z)$ per solid angle of a given class of objects in a given direction are measured. We can consider the number density of galaxies observed at that redshift by assuming that galaxies of a given type are homogeneously distributed in the universe and that they do not appear and disappear within the time span of interest. For each interval $[z ; z+d z]$ of redshift, we count how many galaxies of that kind we see with a redshift in that interval, say $n(z) d z$. Suppose a star or galaxy emits a light ray at time $t_{e}$ and there is an observer located at the origin of our coordinate system where the light arrives at time $t=0$. This means that the emitted light starts at $r\left(t_{e}\right)$ and travels towards the origin $r\left(t_{0}\right)=0$. Considering light-like events (null geodesics) for the Robertson-walker metric in spherical coordinates and using equation (6), it has been shown that [7]:

$$
\int_{R\left(t_{e}\right)}^{R\left(t_{0}\right)} \frac{c d R}{\sqrt{R} \sqrt{\frac{B c^{2} \alpha}{3}-4 \kappa c^{2} R}}=-\int_{r\left(t_{e}\right)}^{r\left(t_{0}\right)} \frac{d r}{1+\kappa r^{2}}
$$




$$
r\left(t_{e}\right)= \begin{cases}\sqrt{\frac{12 R\left(t_{0}\right)}{B c^{2} \alpha}}-\sqrt{\frac{12 R\left(t_{0}\right)}{B c^{2} \alpha(1+z)}}, & \kappa=0 \\ \frac{\sqrt{12 R\left(t_{0}\right)}\left[\sqrt{B c^{2} \alpha(1+z)-12 R\left(t_{0}\right)}-\sqrt{B c^{2} \alpha-12 R\left(t_{0}\right)}\right]}{\sqrt{B c^{2} \alpha-12 R\left(t_{0}\right)} \sqrt{B c^{2} \alpha(1+z)-12 R\left(t_{0}\right)}+12 R\left(t_{0}\right)}, & \kappa=1 \\ \frac{\sqrt{12 R\left(t_{0}\right)}\left[\sqrt{B c^{2} \alpha(1+z)+12 R\left(t_{0}\right)}-\sqrt{B c^{2} \alpha+12 R\left(t_{0}\right)}\right]}{\sqrt{B c^{2} \alpha+12 R\left(t_{0}\right)} \sqrt{B c^{2} \alpha(1+z)+12 R\left(t_{0}\right)}-12 R\left(t_{0}\right)}, & \kappa=-1\end{cases}
$$

and

$$
r(z)=\frac{\sqrt{12 R(t)_{0}}\left[\sqrt{B c^{2} \alpha(1+z)-12 R\left(t_{0}\right)}-\sqrt{B c^{2} \alpha-12 R\left(t_{0}\right)}\right]}{\sqrt{B c^{2} \alpha-12 R\left(t_{0}\right)} \sqrt{B c^{2} \alpha(1+z)-12 R\left(t_{0}\right)}+12 R\left(t_{0}\right)}
$$

where $\kappa=0,1,-1$ is flat, closed and open universe respectively. Differentiating equation (9) w.r.t $z$ and simplifying, we obtain:

$$
\frac{d r}{d z}=\frac{\left(B c^{2} \alpha\right)^{2} \sqrt{3 R\left(t_{0}\right)}}{\sqrt{B c^{2} \alpha-12 R\left(t_{0}\right)} \sqrt{B c^{2} \alpha(1+z)-12 R\left(t_{0}\right)}+12 R\left(t_{0}\right)}
$$

Let $N$ denote the number of galaxies per unit volume of the space with metric

$$
\frac{d r^{2}+r^{2} d \theta^{2}+r^{2} \sin ^{2} \theta d \varphi^{2}}{\left(1+\kappa r^{2}\right)^{2}}
$$

on which the time slices are modeled. The volume element is $r^{2} \sin \theta d \varphi d r /\left(1+\kappa r^{2}\right)^{3}$ hence the number of galaxies between $r$ and $d r$ is given by

$$
\frac{4 \pi r^{2} d r}{\left(1+\kappa r^{2}\right)^{3}} N
$$

Our assumption of the number of galaxies enclosed between the coordinate hyperspheres $r(z)$ and $r(z+d z)$ is therefore given by:

$$
n(z) d z=\frac{4 \pi r(z)^{2} N r^{\prime}(z) d z}{\left(1+\kappa r(z)^{2}\right)^{3}}
$$

where $r^{\prime}(z)=d r / d z$. If we define

$$
\beta_{1}:=B c^{2} \alpha-12 \kappa R\left(t_{0}\right) ; \quad \beta_{2}:=B c^{2} \alpha(1+z)-12 \kappa R\left(t_{0}\right)
$$

then, on substituting equation (9) and (10) into equation (13), we obtain

$$
n(z)=\frac{48 \pi N R\left(t_{0}\right)\left(B c^{2} \alpha\right)^{2} \sqrt{3 R\left(t_{0}\right)}\left[\frac{\sqrt{\beta_{2}}-\sqrt{\beta_{1}}}{\sqrt{\beta_{1} \beta_{2}}+12 \kappa R\left(t_{0}\right)}\right]^{2}}{\left[1+\kappa\left(\frac{\sqrt{\beta_{2}-\beta_{1}}}{\sqrt{\beta_{1} \beta_{2}+12 \kappa R\left(t_{0}\right)}}\right)^{2}\right]^{3}\left[\sqrt{\beta_{1} \beta_{2}}+12 \kappa R\left(t_{0}\right)\right]^{2}}
$$

where $B, \alpha$ and $\beta_{1}, \beta_{2}$ are as defined in equations (5), (6) and (14). Equation (15) gives us the variation of the number density of galaxies with redshift in terms of the cosmic scale factor and mass density of the universe at any

given observational time. This is the principal result in this paper.

Trying to fit the function $n(z)$ to the corresponding counts obtained from observational data by an appropriate choice of parameter values for $R\left(t_{0}\right), \quad \kappa$ and $h\left(t_{0}\right)$, we can tell if our universe is fractal $(D<3)$ or homogeneous $(D=3)$ depending on the value of $D$ for our experimental curve that corresponds to our theoretical curve. It should also be possible to judge whether the observations are compatible with a uniform density of visible matter at some scale in a Friedmann universe depending on the value $D$. Consequently, we should be in position to ascertain if our universe is open, closed or flat depending on the value of $\kappa$. The details of this and computer simulations will constitute our next task.

\section{DISCUSSION AND SUMMARY}

The three-dimensional maps revealing a non-homogeneous universe cast doubt to the validity of the cosmological principle and the Friedmann universe at large. These maps, directly or indirectly, depend on our ability to measure distances. In explict or implicit form, the assumption that the universe is Friedmann may enter into these measurements.

In this paper, we have considered two astronomical quantities (redshift and number density of galaxies) that we have assumed do not involve assumptions about the background geometry. We have established the inter-relationship between the two quantities (equation (15)) by solving the associated Einstein field equations of the universe based on the Friedmann metric. 
Nevertheless, to satisfactorily solve this problem, one needs to perform some computer simulations based on this analytical results and prepare the outcome in a form suitable for comparison with observed dependencies. Therefore, in the work to follow, we shall search for appropriate choice of $\square\left(\square_{0}\right)$, $\square$ and $\square\left(\square_{0}\right)$ while fixing other parameter terms in equation (15) so that we can constrain the relation between $\square$ ( $\square$ ) and $\square$ through computer simulations. Consequently, we shall put our theoretical results in a form suitable for comparison with future observed dependencies that do not involve distance measurements, either explicitly or implicitly, for determining the nature of the universe in question, i.e., whether it is flat, closed or open, depending on the values of $\square$.

\section{ACKNOWLEDGEMENTS}

I would like to thank University of Nairobi for providing me with resource materials and conducive work environment while writing this paper.

\section{REFERENCE}

1. Wilkinson, D., 1998. The Microscope Background Anisotropies: Observations, Proceedings of the National Academy of Sciences of the United of America, 95,1(1998), 29-34.

2. Michal, B. V., 1989. Principles of Cosmology and Gravitation. Institute of Physics Publishing, Bristol.

3. Pietronero, I., Labini, F. S., Montuori, M. 1995. The Debate of Galaxy Correlations and its Theoretical Implications, (arXiv:astro-phy/9510027).

4. Joyce, M., Sylos, L. F., Gabriel, A., Montuori, M., Pietronero, L. 2005. Basic properties of galaxy clustering in the light of recent results from the Sloan Digital Sky Survey, A\&A, 443, 11-16

5. Richard, G., 2012. Advancing the Physics of Cosmic Distances: Conference Summary, Proceedings IAU Symbossium No.289 (arXiv:1209.6529v1, doi:10.1017/S1743921312021709).

6. Potashov, M., Blinnikov, S., Baklanov1, P., Dolgov1, A., 2012. Direct Distance Measurements to SN 2009ip. M NRAS, $000,1-10$

7. Wamalwa, D.S., 2016. On the Friedmann Cosmology, International Journal of Pure and Applied Mathematics, 107, 4, 803-818. doi:10.12732/ijpam.v107i4.2

8. Sylos, F. L., Montuori, M., Pietronero, L. 1998. Scale-invariance of Galaxy Clustering, Physics report, 293, 2, 61226

9. Marcelo, R.B. 1992. On Modelling a Relativistic Hierarchical Model (Fractal) Cosmology by Tolman's Spacetime II: Analysis of the Einstein-De Sitter model, ApJ, 388, 29-33

10. Yurij, B., Pekker, T. Fractal Approach to Large Scale Galaxy Distribution (arXiv:astro-ph/0505185v1)

11. Marcelo, B., Alexandre, Y. M., 1998. Fractals and the Distribution of Galaxies, Braz. J. Phys, 28, 2, 132-160

12. Wald, R. M. 1994. General Relativity. University of Chicago Press Ltd., London, 91-102 